\title{
El intercambio automático de información: hacia el nuevo estándar de transparencia fiscal internacional

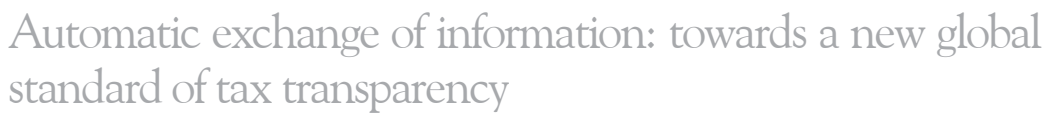

MIGUEL EDUARDO PECHO TRIGUEROS*

Resumen: Las autoridades tributarias dependen cada vez más de la cooperación con sus contrapartes extranjeras para administrar más eficazmente sus leyes tributarias nacionales. Luego de los escándalos bancarios de 2008 y la crisis financiera global posterior, el Foro Global sobre Transparencia e Intercambio de Información Tributaria ha impulsado el intercambio de información a requerimiento como el estándar internacional en materia de transparencia fiscal. Sin embargo, algunas medidas adoptadas por la Unión Europea, iniciativas previas de la Organización para la Cooperación y Desarrollo Económicos (OCDE) y, sobre todo, la introducción en 2010 del «Foreign Account Tax Compliance Act» (FATCA) de los Estados Unidos han promovido la necesidad de adoptar el intercambio automático de información como nuevo estándar de transparencia fiscal internacional. El intercambio automático de información le permite a las jurisdicciones de la residencia verificar si sus contribuyentes han incluido correctamente las rentas obtenidas en el exterior, permitiéndole a las autoridades tributarias contar con alertas tempranas de posibles casos de incumplimiento. En febrero de 2014, la OCDE publicó su propuesta para un nuevo modelo global de intercambio automático de información aplicable a las cuentas mantenidas en entidades financieras. El nuevo modelo global contiene los instrumentos legales necesarios y los procedimientos de debida diligencia y reporte principalmente para las instituciones financieras.

Palabras clave: transparencia fiscal internacional - intercambio automático de información - confidencialidad - Convención Multilateral sobre Asistencia Administrativa Mutua en Materia Fiscal - FATCA - Foro Global sobre Transparencia e Intercambio de Información Tributaria - Países del Grupo de los Ocho (G8) - acceso a la información bancaria con fines tributarios

Abstract: Tax authorities are increasingly relying on mutual cooperation with their foreign peers to enforce more effectively their internal tax laws. After the banking scandals of 2008 and the subsequent global financial crisis, the Global Forum on Transparency and Exchange of Information for Tax Purposes has proposed the exchange of information upon request as the fiscal transparency standard. However, some measures adopted by the European Union, previous initiatives from the Organization for Economic Cooperation

* Director de Estudios e Investigaciones Tributarias del Centro Interamericano de Administraciones Tributarias (CIAT) en Ciudad de Panamá, Panamá. Economista con un MSc en Economía por la University College London. Correo electrónico: mpecho@ ciat.org 
and Development (OECD) and, above all, the introduction of the Foreign Account Tax Compliance Act (FATCA) by the United States in 2010 have promoted the need to adopt the automatic exchange of information as the new fiscal transparency standard. Automatic exchange of information allows home countries to verify whether their taxpayers have correctly included foreign income, allowing tax authorities to have early warning of possible noncompliance cases. In February 2014, the OECD published its proposal for a new global model of automatic exchange of financial account information. The new global model contains the necessary legal instruments and due diligence and reporting procedures, mainly for financial institutions.

Key words: international fiscal transparency - automatic exchange of information - safeguards - Multilateral Convention on Mutual Administrative Assistance in Tax Matters - FATCA - Global Forum on Transparency and Exchange of Information for Tax Purposes - The Group of Eight (G8) - access to bank information for tax purposes

CONTENIDO: I. INTRODUCCIÓN.- II. DESARROLLOS GLOBALES RECIENTES.-
III. EL INTERCAMBIO AUTOMÁTICO DE INFORMACIÓN.- IV. EL MODELO
ÚNICO DE INTERCAMBIO AUTOMÁTICO DE INFORMACIÓN FINANCIERA.

\section{INTRODUCCIÓN}

Las últimas décadas han sido testigo de una liberalización y globalización sin precedentes de las economías. Los países cada vez han eliminado o limitado más los controles sobre las inversiones extranjeras y han disminuido los controles de divisas. Al mismo tiempo, avances en la tecnología de la información y comunicación han hecho que sea más fácil para los inversores ocultar a sus autoridades tributarias las rentas que obtienen en el extranjero.

Mientras que los negocios se hacen globales, las autoridades tributarias permanecen confinadas a sus respectivas jurisdicciones. Así, los funcionarios solo monitorean una parte de las actividades o las inversiones que realiza un contribuyente que opera de forma global. Como resultado, las autoridades tributarias dependen cada vez más de la cooperación con sus contrapartes extranjeras para administrar más eficazmente sus leyes tributarias nacionales ${ }^{1}$.

Un elemento clave de esa cooperación es el intercambio de información. Es una manera eficaz para que los países mantengan la soberanía sobre la aplicación y el cumplimiento de sus leyes tributarias. Los escándalos bancarios de 2008 y la crisis financiera global posterior supusieron un aumento de las preocupaciones de los gobiernos - mayoritariamente de los países de la Organización para la Cooperación y Desarrollo 
Económicos (OCDE) y de la Unión Europea (UE) — por la existencia de déficits fiscales y la fuga de ingresos tributarios, motivando un resurgimiento del interés por el intercambio de información.

El Foro Global sobre Transparencia e Intercambio de Información Tributaria (el Foro Global en adelante) se convirtió entonces en el órgano certificador del estándar internacional en materia de intercambio de información ${ }^{2}$. A través de un proceso de revisiones paritarias, se entiende cumplido el estándar internacional cuando i) existe disponibilidad de información fiscalmente relevante, en particular, de cuentas bancarias y sus titulares; ii) la autoridad tributaria tiene atribuciones para acceder a ella; y iii) la información puede ser intercambiada con otras autoridades tributarias que lo requieran con salvaguardas que protejan los derechos de los contribuyentes y la confidencialidad. Las revisiones evalúan las normativas internas de los países miembros (fase 1) y la aplicación efectiva del intercambio que dichas normas permiten (fase 2). Las revisiones se someten a la asamblea de países y son aprobadas con la regla del consenso menos uno.

Claramente, el estándar internacional promovido por el Foro Global aplica al intercambio de información a requerimiento. Sin embargo, algunas medidas adoptadas por la UE, iniciativas previas de la OCDE y, sobre todo, la introducción en 2010 del «Foreign Account Tax Compliance Act» (FATCA) de los Estados Unidos (EE.UU.) han promovido la adopción del intercambio automático de información como nuevo estándar de transparencia fiscal internacional, con el fin de apoyar a las autoridades tributarias a determinar y recaudar correctamente los impuestos por ingresos del capital obtenidos por sus residentes en el extranjero.

\section{DESARROLLOS GLOBALES RECIENTES}

En julio de 2005 entró en vigencia en la UE la Directiva 2003/48/CE (Directiva sobre la Fiscalidad de los Rendimientos del Ahorro), por la cual los países miembros intercambian automáticamente información sobre los intereses pagados en su jurisdicción a individuos residentes fuera de ella. Luxemburgo y Austria disfrutaron de un período de transición para aplicar esta medida, durante el cual, en lugar de proporcionar información a los demás Estados miembros, aplicaban una retención del $35 \%^{3}$. Para la implementación de la Directiva, el Consejo de la Unión Europea adoptó estándares de reporte común

2 Al momento en que se culminó este artículo, 121 jurisdicciones se habían adherido voluntariamente al Foro Global. Los países de América Latina que participan son Argentina, Brasil, Chile, Colombia, Costa Rica, El Salvador, Guatemala, México, Panamá, República Dominicana y Uruguay.

3 Posteriormente, en abril de 2013, Luxemburgo ha señalado su intención de intercambiar automáticamente información bancaria con otros países miembros de la UE a partir de 2015.

EL INTERCAMBIO AUTOMÁTICO DE INFORMACIÓN: HACIA EL NUEVO ESTÁNDAR DE TRANSPARENCIA FISCAL INTERNACIONAL

AUTOMATIC EXCHANGE OF INFORMATION: TOWARDS A NEW GLOBAL STANDARD OF TAX TRANSPARENCY 
-FISC 39 y FISC 153 - basados en dos trabajos previos de la OCDE: i) el Formato Magnético Estándar (SMF por sus siglas en inglés) de 1997; y ii) el Formato Estándar de Transmisión (STF por sus siglas en inglés) basado en el lenguaje XML, cuya versión más reciente, la 2.1, fue adoptada por los países en 2005. Un proyecto de enmienda de la Directiva que proponía incluir información de personas jurídicas y otras entidades fue rechazado en diciembre de 2013 por el Consejo de Asuntos Económicos y Financieros de la UE .

Otro antecedente del intercambio automático de información es la iniciativa «Treaty Relief and Compliance Enhancement» (TRACE) de la OCDE, que nació con el objetivo de mejorar el procedimiento por el cual los inversionistas en fondos de inversión y fondos de inversión colectiva podían reclamar beneficios derivados de la aplicación de los tratados tributarios, pero que evolucionó a un completo esquema de intercambio automático de información. En enero de 2009, un grupo piloto de países empezó a desarrollar una propuesta de documentación estandarizada (acuerdos y formularios) a ser usados entre los países de la fuente y los intermediarios financieros e inversionistas participantes.

Pero, iha sido FATCA determinante para impulsar el intercambio automático de información? En marzo de 2010, los EE.UU. aprobaron FATCA con el fin de controlar el cumplimiento tributario de los contribuyentes americanos que disponen de activos financieros y obtienen ingresos de los mismos en el exterior. Carbajo y Porporatto definen FATCA como un complejo sistema de procedimientos de debida diligencia, reporte y retención de impuestos, que afecta principalmente a instituciones financieras extranjeras y algunas entidades no financieras privadas que mantienen contactos con los EE.UU ${ }^{5}$. En América Latina ya han firmado un modelo de acuerdo intergubernamental (IGA por sus siglas en inglés) con EE.UU. tanto Costa Rica como México y, según la Federación Latinoamericana de Bancos (FELABAN), Argentina, Brasil, Chile y Panamá vienen teniendo un diálogo activo con las autoridades estadounidenses.

A partir de julio de 2014, los agentes de retención retendrán en la fuente un 30\% sobre cualquier pago de fuente estadounidense (intereses, dividendos, etcétera) que se haga a instituciones financieras extranjeras, a menos que hubieran acordado previamente reportar información sobre

4 La propuesta consideraba incluir el pago de intereses y otros rendimientos del ahorro a personas jurídicas y otras entidades como fundaciones y fideicomisos, incluir otros pagos equivalentes a los intereses derivados de nuevos productos financieros e incluir a nuevos tipos de fondos de inversión colectiva.

5 CARBajo VAsco, Domingo y Pablo PorPoratto. «Los últimos avances en materia de transparencia e intercambio de información tributaria». Documentos de Trabajo 3 del Centro Interamericano de Administraciones Tributarias. Dirección de Estudios e Investigaciones Tributaria (septiembre 2013). El concepto de institución financiera alcanza a bancos, aseguradoras, depositarias, casas de bolsa, intermediarios y agentes financieros $y$ fondos. 
las cuentas estadounidenses a la autoridad tributaria estadounidense, el «Internal Revenue Service» (IRS) ${ }^{6}$. Las instituciones financieras extranjeras aplicarán retenciones del $30 \%$ a otras que no se adhieran e incluso cerrarán cuentas de clientes que no estén de acuerdo con brindar su identificación.

Reconociendo los problemas asociados al carácter extraterritorial de la medida, en junio de 2012, los EE.UU. junto con los países del Grupo de los Cinco (G5) -Alemania, España, Francia, Italia y el Reino Unido- emitieron una declaración conjunta y acordaron firmar un IGA, bajo el cual las instituciones financieras extranjeras cumplirán con sus obligaciones de FATCA reportando a sus respectivas autoridades tributarias la información de las cuentas estadounidenses, la misma que luego será intercambiada automáticamente entre los gobiernos.

Así, los IGA no solo implementarán FATCA, sino que se han convertido en una referencia para avanzar hacia un modelo común de intercambio automático de información. Los países antes mencionados señalaron su compromiso de trabajar junto con la OCDE, la UE y cualquier otro país interesado para adaptar los términos del IGA y convertirlo en el modelo común de intercambio automático de información, incluyendo el desarrollo de estándares de reporte y sistemas de evaluación. En abril de 2013, el G5 anunció su intención de trabajar en un proyecto piloto de intercambio automático multilateral basado en los IGA firmados e invitó a otras jurisdicciones a unirse al proyecto. A esta iniciativa se han sumado doce países europeos (Bélgica, Dinamarca, Eslovenia, Eslovaquia, Finlandia, Irlanda, los Países Bajos, Polonia, Portugal, Rumania, República Checa y Suecia); las dependencias de la corona británica (la Isla del Hombre, la Isla de Jersey y la Isla de Guernesey) y los territorios británicos de ultramar (las Islas de Gran Caimán, Anguila, Bermuda, las Islas Vírgenes Británicas, Monserrat, Gibraltar y las Islas de Turcos y Caicos); y recientemente México, Noruega y Australia.

Actualmente existe ya una propuesta para ampliar el ámbito de aplicación de la Directiva y así incluir información de dividendos, ganancias de capital, saldos de cuentas, cualquier otro ingreso generado con relación a activos mantenidos en una cuenta financiera y cualquier otro ingreso en relación. Igual que en el caso de FATCA, los países del G5 emitieron una carta a la Comisión Europea en abril de 2013 informando su interés por desarrollar un proyecto piloto de intercambio automático multilateral. Esta iniciativa parece correr en paralelo, aunque la UE se ha apresurado en señalar que estará atenta a los desarrollos en la

6 En ausencia del acuerdo, los beneficios derivados de un tratado tributario deberán ser ejercidos mediante solicitudes de devolución de impuestos. 
implementación de FATCA. En ese sentido, el riesgo de superposición se reduce. Además, los países del G5 han hecho declaraciones públicas con relación a la necesidad de adoptar un estándar único basado en FATCA.

\section{Gráfico 1. IGA Modelo 1 e intercambio automático de información}

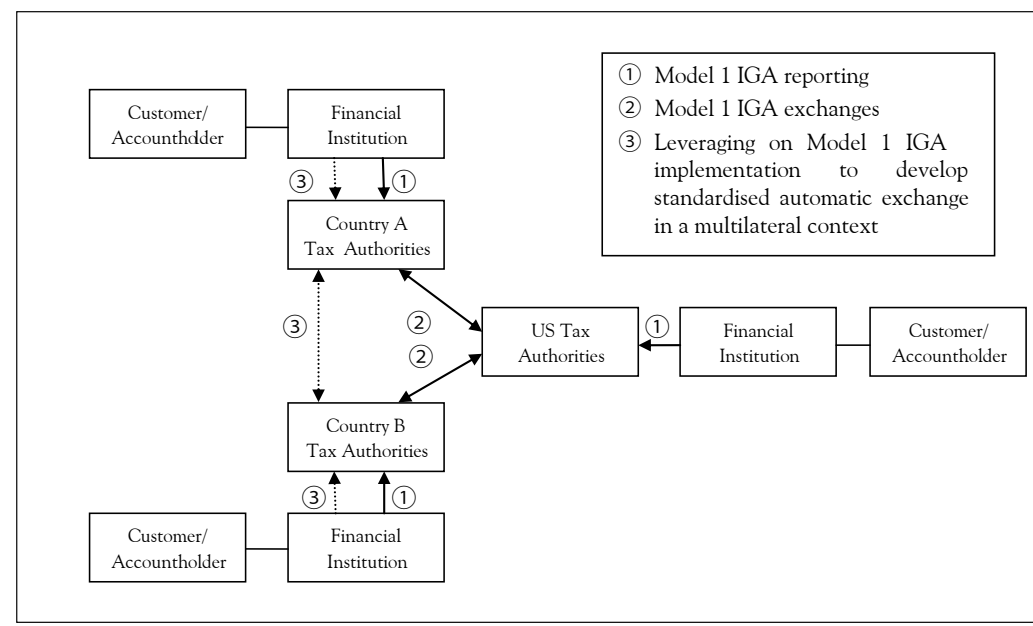

Fuente: OCDE (2013)

\section{EL INTERCAMBIO AUTOMÁTICO DE INFORMACIÓN}

El intercambio automático de información es la transmisión sistemática y periódica de grandes volúmenes de información acerca de las rentas (dividendos, intereses, salarios, entre otros) obtenidas por contribuyentes residentes de una jurisdicción, la cual es obtenida en las jurisdicciones de la fuente de manera rutinaria a través de declaraciones de los pagadores (instituciones financieras, empleadores, entre otros). Son varios los instrumentos legales que permiten a la fecha la implementación de un adecuado intercambio automático de información: i) las disposiciones sobre intercambio de información contenidas en los Convenios para evitar la doble imposición basadas en el artículo 26 tanto del Modelo de Convención de la OCDE como de la Organización de las Naciones Unidas (ONU); ii) el artículo 6 de la Convención Multilateral sobre Asistencia Administrativa Mutua en Materia Fiscal (la Convención Multilateral de aquí en adelante) ${ }^{8}$; iii) para los países miembros de la UE, las leyes domésticas que implementan la Directiva sobre Cooperación

7 OECD. A Step Change in Tax Transparency. Delivering a Standardised, Secure and Cost Effective Model of Bilateral Automatic Exchange for the Multilateral Context. Reporte de la OCDE para la cumbre G8 de junio de 2013.

8 A la fecha son 64 las jurisdicciones firmantes. Los países de América Latina que se han adherido a la Convención Multilateral son Argentina, Brasil, Colombia, Costa Rica, Guatemala y México. Solo en Argentina, Costa Rica y México se encuentra vigente. 
Administrativa en el Ámbito de la Fiscalidad antes mencionada; y iv) los acuerdos bilaterales basados en el modelo de acuerdo de intercambio de informaciones tributarias del CIAT. Cabe mencionar que los países podrán acordar la celebración de Acuerdos Especiales o Memorandos de Entendimiento para establecer los términos y condiciones del intercambio automático acordado.

El intercambio automático de información le permite a las jurisdicciones de la residencia verificar si sus contribuyentes han incluido correctamente las rentas obtenidas en el exterior en países relacionados de la misma región geográfica, socios comerciales o países donde sus contribuyentes usualmente invierten. Además, le permite a las autoridades tributarias contar con alertas tempranas de posibles casos de incumplimiento. Estas pueden luego llevar a cabo programas para identificar a los no declarantes, enviar cartas a los contribuyentes que han omitido declarar sus rentas de fuente extranjera o solicitar datos sobre el origen de los fondos que las generaron, usar los datos recibidos a través del intercambio automático para la programación de auditorías, entre otros. Los datos también pueden ser usados transversalmente por otras agencias de gobierno para identificar, por ejemplo, posibles casos de lavado de dinero. Hay evidencia para países miembros de la OCDE de la utilidad que ha tenido el intercambio automático para identificar esquemas de este tipo en el sector inmobiliario. Sirve además de mecanismo de disuasión. Pueden usarse adecuadas estrategias de comunicación sobre el intercambio automático de información para transmitir mensajes claves a los contribuyentes y así concientizarlos. En el material de orientación pueden incluirse referencias a las posibilidades de intercambio con otros países que puede ejercer la autoridad tributaria donde residen. Al final de cuentas, estas acciones tienen un impacto positivo en el cumplimiento voluntario y, por supuesto, en los recaudos y la justicia del sistema tributario.

El intercambio automático de información requiere trabajar para que la información sea capturada, intercambiada y procesada rápida y eficientemente por la jurisdicción receptora. Además, como ya se mencionó, deben usarse métodos seguros y compatibles de transmisión y encriptación de datos desde las instituciones que proveen la información a las autoridades tributarias nacionales y desde estas últimas a las autoridades tributarias extranjeras. En ese sentido, son requeridos métodos de transmisión y codificación compatibles, herramientas de codificación y descodificación de datos, espacios de almacenamiento de datos, herramientas de tratamiento de datos tales como los de coincidencia y conversión de divisas. Pero, por encima de todo, la transmisión y almacenamiento de la información debe ser totalmente segura y muy confidencial. Como se ha mencionado anteriormente, 
dos aportes previos de la OCDE —el SMF y el STF_ pueden tomarse como referencia para lograr estos objetivos.

No menos importantes son las herramientas de explotación de los datos. Para poder tomar ventaja del intercambio automático de información, los funcionarios deben ser capacitados para integrar la información recibida en sus procesos de auditoría fiscal, para asegurar que los datos sean clasificados e ingresados correctamente, lo que podría suponer controles manuales, para utilizar para otros fines la información intercambiada previo acuerdo del país que la envía e identificar las oportunidades para la cooperación con otras agencias de gobierno. Es recomendable por ello diseñar programas especiales que faciliten la minería de datos multidimensional (por dirección, transacción, ordenante, receptor, etcétera). Una referencia es la plataforma «Foreign Automatic Information Retrieval System» (FAIRS) de Canadá.

Finalmente, el intercambio automático de información requiere tomar en consideración algunos aspectos institucionales. Las jurisdicciones deben ser capaces de obtener la información, de poder intercambiarla y, por supuesto, de salvaguardarla. Por ello, debe dotarse a las autoridades competentes de la infraestructura necesaria para poder implementar efectivamente el intercambio automático (suficiente capacidad de almacenamiento de datos, seguridad de los sistemas de información), dotarlas de un número suficiente de funcionarios debidamente capacitados, así como facilitarles un adecuado canal de comunicación con sus pares en el extranjero.

En América Latina los asuntos de la tributación internacional han llamado el interés de las autoridades tributarias solo recientemente. Un estudio de CIAT/BID/CAPTAC-DR, señalaba que antes del año 2008 solo en la mitad de los países de la región las autoridades tributarias habían implementado unidades a cargo de la administración de los tratados tributarios, el control de la manipulación de los precios de transferencia y el intercambio de información. Parece que deberá pasar algún tiempo más para que el resto de países tomen conciencia de las consecuencias de no controlar adecuadamente las transacciones transfronterizas de los contribuyentes. 
Cuadro 1. Unidades a cargo de asuntos de la tributación internacional en las autoridades tributarias de América Latina ${ }^{9}$

\begin{tabular}{|l|c|c|}
\hline & Creada antes de 2008 & Creada después de 2008 \\
\hline Argentina (AFIP) & Sí \\
\hline Bolivia (SIN) & - & - \\
\hline Brasil (RFB) & Sí & - \\
\hline Chile (SII) & Sí & - \\
\hline Colombia (DIAN) & Sí & - \\
\hline Costa Rica (DGT) & - & - \\
\hline Ecuador (SRI) & Sí & - \\
\hline El Salvador (DGI) & - & - \\
\hline Guatemala (SAT) & - & - \\
\hline Honduras (DEI) & - & - \\
\hline México (SAT) & Sí & - \\
\hline Nicaragua (DGI) & - & Sí \\
\hline Panamá (DGI) & Sí & Sí \\
\hline Paraguay (SET) & - & Sí \\
\hline Perú (SuNAT) & - & - \\
\hline República Dominicana & - & \\
\hline Uruguay (DGI) & & - \\
\hline Venezuela (SENIAT) & & - \\
\hline
\end{tabular}

EL INTERCAMBIO

AUTOMÁTICO DE

INFORMACIÓN:

HACIA EL NUEVO

ESTÁNDAR DE

TRANSPARENCIA

FISCAL INTERNA-

CIONAL

\section{AUTOMATIC}

EXCHANGE OF

INFORMATION:

TOWARDS A NEW

GLOBAL STAN-

DARD OF TAX

TRANSPARENCY

Es muy importante, en esta etapa del intercambio automático de información, apoyar a los países en desarrollo o en transición con programas de construcción de capacidades y asistencias técnicas. El rol del Foro Global y los organismos internacionales y regionales es fundamental para prestar la ayuda necesaria a los países que aún se encuentran rezagados. El Foro Global ha creado un subgrupo de trabajo que tiene a su cargo la identificación de las principales dificultades que enfrentan los países para implementar adecuadamente el intercambio automático de información. Este subgrupo lo conforman 51 países

9 CIAT/BID/CAPTAC-DR. «Estado de la Administración Tributaria en América Latina: 2006-2010», 2012. 
miembros. Argentina, Brasil, Colombia (vicepresidente del subgrupo) y México son los países que representan a América Latina.

La ONU ha comenzado a discutir en el seno de su Comité de Expertos sobre Cooperación Internacional en Cuestiones de Tributación (el Comité de Expertos en adelante) cómo apoyar la adopción del intercambio automático de información como nuevo estándar global. De momento, el Comité de Expertos está recomendando a los países la firma de la Convención Multilateral y apoyando actividades de construcción de capacidades. En su más reciente reunión en Ginebra, los miembros del Comité de Expertos pidieron estudios más profundos acerca de los asuntos de confidencialidad que implica el intercambio automático de información, no sin antes reconocer las limitaciones de recursos y dificultades prácticas que enfrentarán las autoridades tributarias de los países menos desarrollados para implementar correctamente el nuevo estándar.

\section{EL MODELO ÚNICO DE INTERCAMBIO AUTOMÁTICO DE INFORMACIÓN FINANCIERA}

Además de recomendar reiteradamente a los países su adhesión a la Convención Multilateral, los líderes de los países del Grupo de los Ocho (G8) y del Grupo de los Veinte (G20) han brindado su total apoyo a la adopción del intercambio automático de información como nuevo estándar de transparencia fiscal internacional. En 2013, ante una solicitud de la presidencia del G8 -el Reino Unido-, la OCDE presentó recomendaciones de cómo avanzar hacia un estándar universal de intercambio automático de información. Básicamente recomendó trabajar en cuatro medidas para una correcta implementación: i) la promulgación de un marco legal amplio a fin de facilitar la expansión de las jurisdicciones asociadas; (ii) seleccionar una base jurídica para el intercambio de información; (iii) adaptar el alcance de los requisitos de debida diligencia y guías de coordinación; y (iv) el desarrollo de estándares compatibles o comunes de tecnologías de información y comunicaciones. Los líderes del G8 hicieron suyo el informe en junio de $2013^{10}$. Igualmente los líderes del G20, reunidos en San Petersburgo, apoyaron plenamente la propuesta de la OCDE de un modelo global de intercambio automático de información, comprometiéndose a convertirlo en el nuevo estándar mundial, esperando iniciar el intercambio a finales de $2015^{11}$.

10 OECD. A Step Change in Tax Transparency. Delivering a Standardised, Secure and Cost Effective Model of Bilateral Automatic Exchange for the Multilateral Context.

11 OECD. Standard for Automatic Exchange of Financial Account Information: Common Reporting Standard, Reporte presentado al G20 en San Petersburgo, 2014. 
El 13 de febrero de 2014, la OCDE finalmente publicó su propuesta de nuevo modelo global aplicable a las cuentas mantenidas en entidades financieras. El nuevo modelo tiene dos componentes: un Acuerdo entre Autoridades Competentes (CAA por sus siglas en inglés) y el Estándar Común de Información (CRS por sus siglas en inglés). El primero es un modelo de acuerdo que las jurisdicciones que deseen implementar el nuevo estándar global de intercambio automático de información deberán firmar. El segundo es el conjunto de reglas mínimas comunes con relación al reporte de la información y la debida diligencia que deberán llevar a cabo las instituciones financieras.

El modelo CAA toma en cuenta los CRS y los instrumentos legales que permiten el intercambio (los tratados o acuerdos bilaterales y la Convención Multilateral antes mencionados). Contiene una serie de considerandos y siete secciones. Los considerandos incluyen aseveraciones respecto a las reglas domésticas sobre presentación de reportes y debida diligencia que apuntalan el intercambio de información conforme al CAA. También incluyen aseveraciones sobre confidencialidad, salvaguardas y la existencia de la infraestructura necesaria para una efectiva relación de intercambio. Las secciones definen el ámbito de aplicación, el tiempo y la forma del intercambio, la confidencialidad y la salvaguarda de los datos que deben ser respetados, y la forma cómo lidiar con las consultas entre autoridades competentes, las modificaciones o la terminación del acuerdo, entre otros. El CAA está pensado para un intercambio recíproco, pero, al igual que el IGA tipo FATCA, puede adaptarse a una versión no recíproca.

El CRS describe los estándares de reporte y debida diligencia que implementa el intercambio automático de información de cuentas en entidades financieras, en la misma línea que los IGA tipo FATCA. Las instituciones financieras consideradas como obligadas a seguir el CRS son los bancos, las instituciones de custodia y las instituciones de depósito, pero también ciertos vehículos colectivos de inversión y ciertas compañías de seguro. La información a reportar incluye los intereses, los dividendos y los rendimientos de ciertos contratos de seguro, pero también los saldos de cuentas y los resultados de la venta de activos financieros. El espectro de reporte incluye las cuentas mantenidas por individuos y entidades (como fideicomisos y fundaciones) y las cuentas mantenidas por entidades pasivas no financieras con el fin de alcanzar a las personas que las controlan. Es de esperarse que cada país especifique los tipos de entidades que quedarían exentas de la obligación de seguir el CRS, al igual que lo experimentado por los IGA tipo FATCA.

En cuanto a los procedimientos de debida diligencia que deberán llevar a cabo las instituciones financieras, se establecen diferencias sea que se trate de cuentas individuales o de entidades; o que se trate de cuentas 
nuevas o preexistentes, puesto que será más difícil para las instituciones financieras obtener información de los titulares de estas últimas. Los umbrales solo se fijan para las cuentas de entidades. Para las cuentas preexistentes de individuos, las instituciones financieras deberán revisar todas las cuentas, aunque serán más rigurosas para la cuentas de más alto valor. Para las cuentas nuevas, la CRS contempla un procedimiento de autocertificación como parte de sus procedimientos de apertura de cuentas, que le permita determinar su residencia para propósitos tributarios, incluyendo cualquier documentación recopilada como parte de los procedimientos «Conoce a tu cliente» o de lucha contra el lavado de dinero. Para las cuentas preexistentes de entidades, las instituciones financieras solo están obligadas a hacer la revisión si estas mantienen saldos superiores a US\$250 000 (o su equivalente). Para determinar si la cuenta es susceptible de ser reportada, se deberá verificar la residencia del titular, sobre la base de cualquier documentación recopilada como parte de los procedimientos «Conoce a tu cliente» o de lucha contra el lavado de dinero, o en todo caso por algún procedimiento de autocertificación. El mismo procedimiento aplica para las cuentas nuevas de entidades, aunque como es más fácil aplicar un procedimiento de autocertificación, el umbral de US\$250 000 no aplica.

Cabe resaltar que el nuevo estándar global de intercambio automático de información no contempla ningún tipo de retención como la establecida por FATCA. Asimismo, no reemplaza el estándar de intercambio a requerimiento, simplemente lo complementa. Sobre este último punto, dada la potencia del intercambio automático de información, no sería raro que cuando el nuevo estándar sea adoptado mundialmente, lo que termine siendo complementario sea el intercambio de información a requerimiento.

El reto ahora es lograr que la recomendación sea adoptada globalmente. Al menos los cuarenta países que vienen trabajando en las pruebas piloto lo harán suyo inmediatamente. De igual forma, se espera que lo hagan suyo los líderes del G20. En ese sentido, se apreciará una carrera por la firma de CAA en el transcurso de los años 2014 y 2015, llevando a una implementación efectiva del intercambio automático de información probablemente a partir de 2016.

Para los países que lo adopten, el CRS se convertirá en el punto de partida de cualquier negociación para la firma de un CAA. Fijará los procedimientos de debida diligencia a seguir y conducirá a los países a adoptar cambios en su legislación interna para levantar las restricciones de acceso a la información bancaria a efectos de cumplir con el intercambio automático de información o permitir que las instituciones financieras puedan recopilar más información de los clientes y transmitirla a las autoridades tributarias. 
Debe notarse que hay aún muchas jurisdicciones de Asia, América Latina y Medio Oriente que no participan del proyecto piloto. En ese sentido, el reto es grande para el Foro Global, el cual se encargará de monitorear la adopción del nuevo estándar a nivel mundial. Un incentivo para que más países hagan suyo el nuevo modelo será, sin duda, la efectividad que muestre la medida para incrementar el cumplimiento voluntario.

\section{Cuadro 2. Acceso a la información bancaria con fines tributarios en América Latina ${ }^{12}$}

\begin{tabular}{|l|c|}
\hline Argentina & Sí \\
\hline Bolivia & No \\
\hline Brasil & Sí (2009) \\
\hline Chile & Sí \\
\hline Colombia & No \\
\hline Costa Rica & Sí (2009) \\
\hline Ecuador & No \\
\hline El Salvador & No \\
\hline Guatemala & No \\
\hline Honduras & Sí \\
\hline México & No \\
\hline Nicaragua & Sí (2010) \\
\hline Panamá & No \\
\hline Paraguay & No \\
\hline Perú & No \\
\hline República Dominicana & \\
\hline Uruguay & \\
\hline
\end{tabular}

EL INTERCAMBIO

AUTOMÁTICO DE

INFORMACIÓN:

HACIA EL NUEVO

ESTÁNDAR DE

TRANSPARENCIA

FISCAL INTERNA-

CIONAL

AUTOMATIC

EXCHANGE OF

INFORMATION:

TOWARDS A NEW

GLOBAL STAN-

DARD OF TAX

TRANSPARENCY

Pero también será un incentivo el avance que se registre en materia de acceso a la información bancaria con fines tributarios. En América Latina, por ejemplo, aún es necesario eliminar barreras en la mitad de los países de la región. De nada valdrá que un país firme la Convención Multilateral u otros instrumentos legales si es que las leyes domésticas no se ajustan a este instrumento supranacional para permitir el 
intercambio de la información bancaria, como ocurre en los casos de Costa Rica y Guatemala. Para avanzar hacia una mayor transparencia fiscal en la región, Velayos y Barreix resaltan la necesidad de vencer la resistencia de los grupos de interés, que presionan fuertemente para que no se avance en este terreno, especialmente en los países que son plazas financieras ${ }^{13}$; y la necesidad de que los gobiernos generen un clima de confianza y seguridad para que sus ciudadanos no busquen trasladar su patrimonio hacia el exterior, toda vez que en el pasado la región ha tenido experiencias intervencionistas, de malos manejos de los recursos públicos e inseguridad. 